\title{
Towards a Complex Automata Multiscale Model of In-Stent Restenosis
}

\author{
Alfonso Caiazzo ${ }^{1}$, David Evans ${ }^{2}$, Jean-Luc Falcone ${ }^{3}$, Jan Hegewald ${ }^{4}$, \\ Eric Lorenz ${ }^{5}$, Bernd Stahl ${ }^{3}$, Dinan Wang ${ }^{6}$, \\ Jörg Bernsdorf ${ }^{6}$, Bastien Chopard ${ }^{3}$, Julian Gunn ${ }^{2}$, \\ Rod Hose ${ }^{2}$, Manfred Krafczyk ${ }^{4}$, Patricia Lawford ${ }^{2}$, Rod Smallwood ${ }^{2}$, \\ Dawn Walker ${ }^{2}$, and Alfons G. Hoekstra ${ }^{5}$ \\ 1 INRIA Rocquencourt, France \\ \{alfonso.caiazzo\}@inria.fr \\ 2 The University of Sheffield, UK \\ \{david.evans, j.gunn, d.r.hose,p.lawford,r.smallwood, \\ d.c.walker\}@sheffield.ac.uk \\ ${ }^{3}$ CUI Department, University of Geneva, Switzerland \\ \{jean-luc.falcone, bernd.stahl, bastien.chopard\}@unige.ch \\ ${ }^{4}$ Technical University of Braunschweig, Germany \\ \{hegewald, kraft\}@irmb.tu-bs.de \\ ${ }^{5}$ University of Amsterdam, the Netherlands \\ \{e.lorenz, a.g.hoekstra\}@uva.nl \\ ${ }^{6}$ NEC Laboratories Europe, St. Augustin, Germany \\ $\{$ d. wang, $j$. bernsdorf $\} @ i t . n e c l a b . e u$
}

\begin{abstract}
In-stent restenosis, the maladaptive response of a blood vessel to injury caused by the deployment of a stent, is a multiscale problem involving a large number of processes. We describe a Complex Automata Model for in-stent restenosis, coupling a bulk flow, drug diffusion, and smooth muscle cell model, all operating on different time scales. Details of the single scale models and of the coupling interfaces are described, together with first simulation results, obtained with a dedicated software environment for Complex Automata simulations. The results show that the model can reproduce growth trends observed in experimental studies.
\end{abstract}

Keywords: Complex Automata, in-stent restenosis, multiscale modeling.

\section{Introduction}

A stenosis is a narrowing of a blood vessel lumen due to the presence of an atherosclerotic plaque. This can be corrected by balloon angioplasty, after which a stent (metal mesh) is deployed to prevent the vessel from collapsing. The injury caused by the stent can lead to a maladaptive biological response of the cellular tissue (mainly smooth muscle cells). The abnormal growth can produce a new stenosis (re-stenosis). The multiscale nature of this process has been discussed in detail previously by Evans et al. [1]. 
The geometry of the stent employed influences the biological events occurring in the vessel following deployment. Stent length, strut thickness, number, crosssectional shape and arrangement all influence the hemodynamics and degree of injury/stretch observed within the stented segment [2]. These in turn, are critical determinants of the severity of restenosis observed. Additionally, stents may be coated with active compounds targeted at the biological processes responsible for driving the progression of a restenosis which, when eluted locally at the stented site, can prevent proliferation of smooth muscle cells and neointimal growth.

The development of a multiscale in silico model capable of testing both the influence of stent geometry and that of drug elution is motivated by the desire for a better understanding of the dynamics regulating restenosis. Thus providing a potentially powerful tool for improved understanding of the biology, and to assist in the process of device/therapy development.

As in many other biological systems, the dynamics of in-stent restenosis span many orders of magnitude through the scales, from the smallest microscopic scales up to the largest macroscopic ones. The wealth of experimental data that is now available has made in silico experimentation an attractive tool in systems biology, allowing hypothesis testing and formulation of predictions which can be further tested in vitro or in vivo 3 . The next challenge is to study, not only fundamental processes, on all these separate scales, but also their mutual coupling across the scales and to determine the emergent structure and function of the resulting system [4].

Based on the conceptual description of the relevant processes and their characteristic scales as presented in [1, we propose a simplified CxA model of in-stent restenosis, coupling a lattice Boltzmann bulk flow (BF) solver (for the blood flow), an agent based model for smooth muscle cell (SMC) dynamics (simulating growth, cell cycle, physical and biological cell-cell interaction), and a Finite Difference scheme for the drug diffusion (DD) within the cellular tissue.

In section 2 we introduce the main ideas behind the CxA approach. In section 3 following a short introduction on instent restenosis, we present the multiscale model. We describe the main characteristics of the single scale solvers, which have been developed independently from one another, and independently from the ultimate application. Then, in section 4 we discuss how the coupling between these models has been realized using a $\mathrm{CxA}$ dedicated software environment. Preliminary numerical results are presented in section 5, and conclusions are discussed in section 6 .

\section{Complex Automata Modeling and Simulation}

Recently we introduced Complex Automata (CxA) as a paradigm for multiscale modelling and simulation 578 . A multiscale system is decomposed into mutually interacting single scale models. The multiscale system can be represented graphically on a Scale Separation Map (SSM), where the horizontal and vertical axes represent the temporal and spatial scales. An example of such SSM is shown in Fig. 1 for the multiscale model of in-stent restenosis (as discussed in 
detail in section (3). The single scale models and their interactions are drawn on the map. The single scale models are discrete and explicitly update their state to a next time step using a well defined evolution operator, in the form of collision+propagation 1. An essential step in the modelling process is the inclusion of specific coupling templates, designed to mimic the dynamic behavior of the multi-scale process as accurately as possible.

The conceptual ideas behind the CxA approach have been used to develop the COAST Multiscale Coupling Library and Environment (MUSCLE) [112], a software environment where a $\mathrm{CxA}$ can be implemented naturally. The coupling library is based on JADE, a multiagent platform, where both the kernels (i.e. the single scale models) and the conduits (i.e. the multiscale couplings) are treated as agents. The single scale models do not need to be aware of each other, and the information concerning the coupling and the global setup are held by the framework. The structure of the coupling library allows a complete independence from native codes, which can be replaced with a different source, provided the interface with respect to the framework (i.e. the wrapper agent) remains the same. Kernels communicate via smart conduits (special agents which implement the multiscale coupling) using two communication primitives, a non-blocking send operation and a blocking receive operation. Often, conduits perform filtering operations, in order to match the input and output of the different single scale models. The details of coupling for this particular application are discussed in section 4 .

\section{Multiscale Model of In-Stent Restenosis}

In a previous work [1, the processes key to the restenosis system and their mutual coupling were identified and considered in terms of their temporal and spatial scales, allowing us to generate a comprehensive Scale Separation Map [1]. In this paper, we consider a simplified model focused on SMC behavior, and the interaction with blood flow and drug eluted from the stent. A simplified SSM is shown in Fig. 1. After deployment of the stent, which is actually modelled in a separate box as an initial condition (using the SMC model itself, see section 4), SMCs start to proliferate. This proliferation depends on the blood flow (specifically the wall shear stress) and the concentration of drug (in the case of a drug eluting stent). The blood flow, in turn, depends on the lumen domain (and therefore changes due to the proliferation of SMC), and the concentration of drug depends on the SMC domain itself (and therefore also on SMC proliferation).

In the current model we assume that scale separation between the single scale models is confined to the temporal scale. The SMC proliferation is the slowest process, dictated by the cell cycle whereas flow is a fast process, dictated by one heart beat. Due to the specific value of the diffusion coefficients and the typical size of the tissue level, the temporal scale of the diffusion process resides between

1 The terminology collision-propagation is borrowed from the lattice gas automata framework (see e.g. 9]), and it has been recently shown to be equivalent to other update paradigms 10 . 


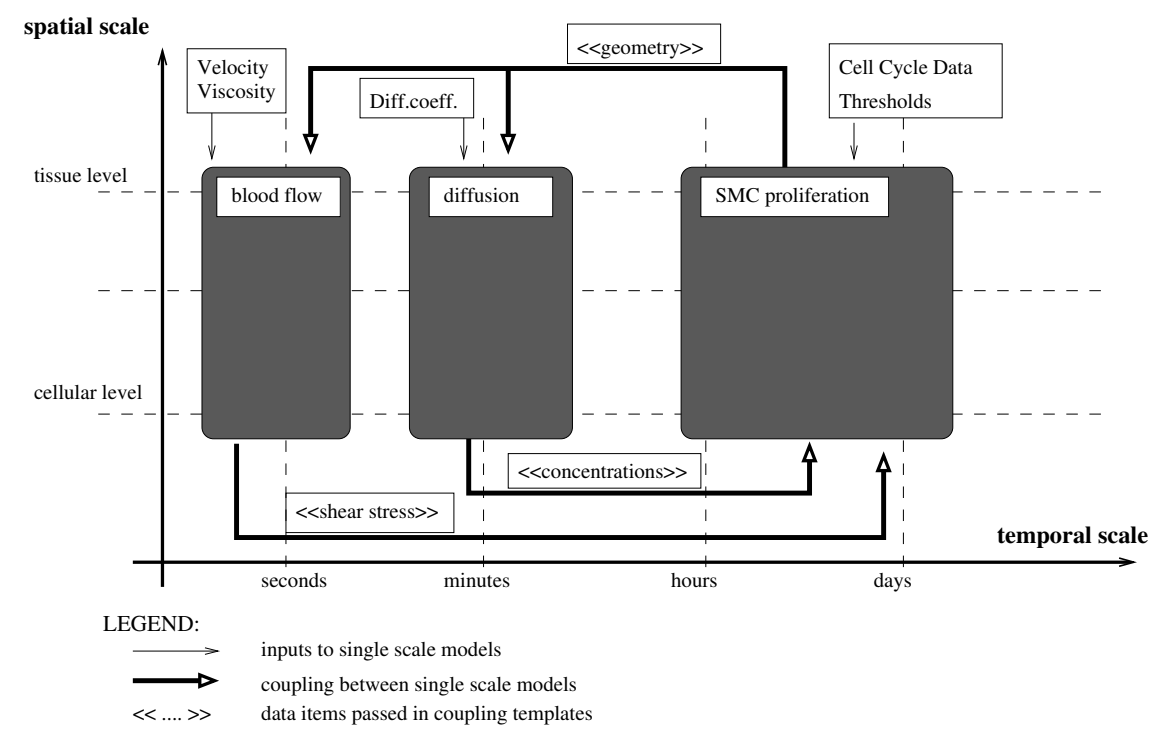

Fig. 1. The simplified SSM

the flow and the SMC scales. In future models we will also explicitly consider spatial scale separation.

The blood flow inside the vessel is modeled as a Newtonian incompressible flow. We employ a lattice Boltzmann Method to obtain a numerical solution (see for example 913]). The observable related to the bulk flow (BF) model is the wall shear stress on the vessel boundary (WSS). This is needed as input for the SMC model, after being properly mapped (section 4).

The dynamics of smooth muscle cells are simulated using an Agent Based Model (SMC model), where each cell represents a single agent, identified by a set of state-variables: position, radius, biological state, drug concentration and structural stress. Each SMC agent evolves in time according to the current state, and to the states of neighboring cells. The solver comprises a physical solver, simulating the structural dynamics of cells, and a biological solver which simulates the cell cycle, according to a biological rule set. More detail of the SMC model will be published elsewhere [14]. Cells are represented by their centres and potential functions, which determine non-linear repulsive and attractive inter-cell forces. In additional, boundary forces, viscous friction and radial elastic forces modeling the primary fibre direction of real 3D SMCs and motility forces modeling cell migration are taken into account [14. At every iteration step, new equilibrium positions of SMCs are computed by iterating a finite difference scheme until steady state is reached. Next, the structural stress is calculated and provided as input to the biological solver.

The biological solver contains a model of the cell cycle, consisting of a discrete set of states, a quiescent state $\mathbf{G 0}$, a growth state $\mathbf{G} \mathbf{1}$ and finally a mitotic 
state $\mathbf{S} / \mathbf{G 2} / \mathbf{M}$, when a mother cell eventually divides into two new daughter cells. Progression through the cell cycle takes place at a fixed rate, depending on the time step, and culminates in mitosis (division). Cells may enter or leave the inactive phase of the cell cycle (G0) depending on certain rules, which take into account contact inhibition (based on neighbor count), structural stress, and local drug concentration (for all cells) and wall shear stress (WSS) and oscillatory stress index (OSI) for cells in contact with the fluid. Low WSS or high structural strain are individually capable of inducing agent proliferation if drug concentration and contact inhibition criteria allow.

Drug eluting stents represent an effective way of inhibiting neointimal formation. We include this additional aspect in the $\mathrm{CxA}$ model by implementing a drug diffusion sub-process. Since drug is eluted from the stent and diffuses into the cellular tissue, the spatial domain for the Drug Diffusion Model coincides with the space occupied by SMCs. The stent struts act as a source, whilst the boundaries between flow and cells are considered sinks (assuming that drug eluted into the lumen is continuously flushed away by the faster blood flow). Since biological tissues are heterogeneous in nature, we assume that this process can be described using a generic anisotropic diffusion.

The diffusion tensor is chosen such that the diffusion along the artery axis or tangentially to a cross section is at least 10 times higher than the diffusion in the radial direction [1516. To solve the diffusion equation numerically we use a Finite Differences (FD) approach, solved by a Propagation-Collision loop, which complies with the CxA modeling language. According to [16], the time scale to reach the steady state is of the order of minutes. Therefore, when coupling DD and SMC, we are largely interested in the steady drug concentration (the time step for the SMC model, which uses as input the drug concentration, is of the order of 1 hour).

\section{CxA Implementation: Connection Scheme, Kernels, Conduits}

In order to combine the single scale models in a CxA setup, we need to define a communication graph, the Connection Scheme (CS), which specifies in detail the communication topology of the CxA, i.e. which pairs of single scale models, the kernels of the $\mathrm{CxA}$, communicate. For the simplified model of in-stent restenosis, the CS is shown in Figure 2 .

At each SMC iteration, the cell configuration defines a new domain for the BF solver. The conduit from SMC to BF converts the array of positions and radii of the cells into a computational mesh, decomposed into fluid and solid nodes for the flow solver.

Similarly, a conduit from SMC to DD converts the array of positions and radii of the cells, into a computational mesh for the drug diffusion solver, marking the nodes as tissue, source, or sink.

plus .1em In some cases, the interaction between kernels is slightly more complex, and multiple inputs are required for computing an output. This is the case for the 


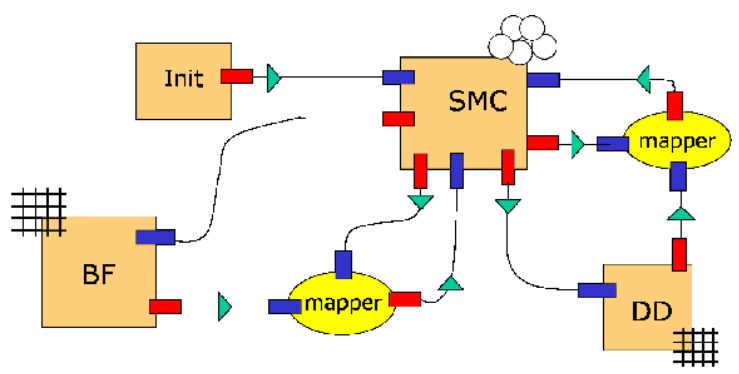

Fig. 2. The Connection Scheme, showing the single scale models (Bulk Flow, SMC, Drug Diffusion), the Init agent (used to generate the initial structural stress condition in the tissue), the mapper agents and the conduits. For each single scale models it is specified whether it is mesh-based (BF, DD) or Agent-based (SMC).

mapper agents in (Fig.22). For example, since the SMC agents are moving, each time an input for the SMC model has to be computed, the SMC itself has to send its current state, to allow the coupling agent to map the values to each agent.

The values of fluid shear stress at the boundary affect the biological evolution of the cells. Given the fluid output, and the current cell configuration, a mapper agent computes the shear stress on each surface cell. Depending on the discretization used in the flow solver, different approaches can be used. If the flow grid is coarser than the spatial scale of the SMC model (for example, the radius of the cells), a proper algorithm must be used in order to determine which cells are in contact with the flow, then for each cell position, the shear stress is extrapolated from the closest boundary fluid nodes. Alternatively, if the flow discretization is sufficiently fine, allowing more fluid boundary nodes to interact with single cells, the shear stress on the cell surface can be calculated by averaging the values of the closest nodes.

Given the current drug concentrations and the cell configuration, the mapper agent approximates the concentration on each cell. As is the case for the shear stress approximation, the algorithm used depends on the grid size of the DD model. If the grid is sufficiently fine, with many lattice nodes per SMC, the concentration on a cell can be integrated. If a coarse DD grid is used, the concentration for each cell will be extrapolated using the closest nodes.

The single scale models, BF, SMC, DD, are implemented in different programming languages (FORTRAN90, C++, JAVA), wrapped as JAVA agents [11/12], and connected as shown in the Connection Scheme (Fig. 2). In the current setup we have 25 agents ( 3 kernels, 20 conduits and 2 mappers) which participate in the main computation of our prototype application. These can be distributed across multiple CPUs and machines to gain an advantage in computing speed.

\section{Simulation Results}

As a benchmark geometry for the 2D CxA model, we consider a vessel, of length $1.5 \mathrm{~mm}$ and width $1.0 \mathrm{~mm}$, where two square struts of side length $90 \mu \mathrm{m}$ have 


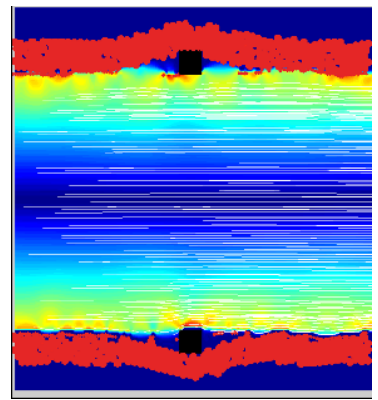

Fig. 3. Initial Condition for the CxA model, including initial cell configuration, equilibrated after deployment, and blood flow (the flow is shown in the lumen as streamlines, and the fluid shear stresses are color coded (red high, blue low)

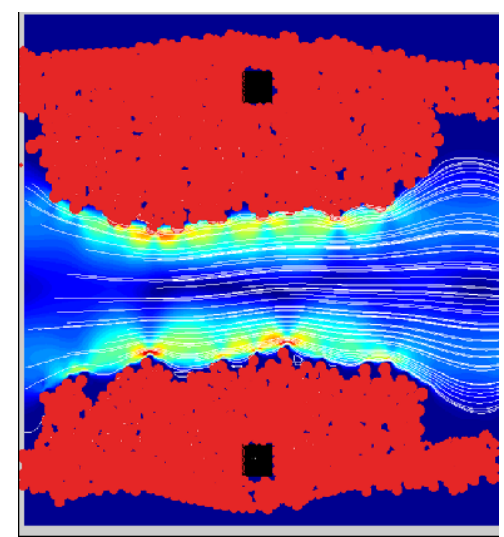

Fig. 4. Results with bare metal stent after 400 time steps ( $\sim 18$ days). The flow is shown in the lumen as stream lines, and the fluid shear stresses are color coded (red high, blue low).

been deployed. The vessel wall has a thickness of $120 \mu \mathrm{m}$. Smooth muscle cells are generated with an average radius of $15 \mu \mathrm{m}$, densely packed inside the wall. To obtain the initial conditions based on the above geometry, an initial stress configuration compatible with the initial geometry must be provided. To do this, cells are stent deployment is simulated, iterating the structural SMC solver until a stationary state is reached. The initial cell configuration resulting from this procedure is shown in Fig. 3. The struts are shown in black, the SMC in red.

We have run the simulation for 400 time steps of the SMC model $(\Delta t=1$ hour) for both a bare metal stent and a drug eluting stent. The final results are shown in Fig. 4 and 5 respectively. The preliminary results of the model demonstrate smooth muscle cell proliferation in response to injury caused by the stent deployment. Proliferation is modulated by flow, local mechanical stress, and 


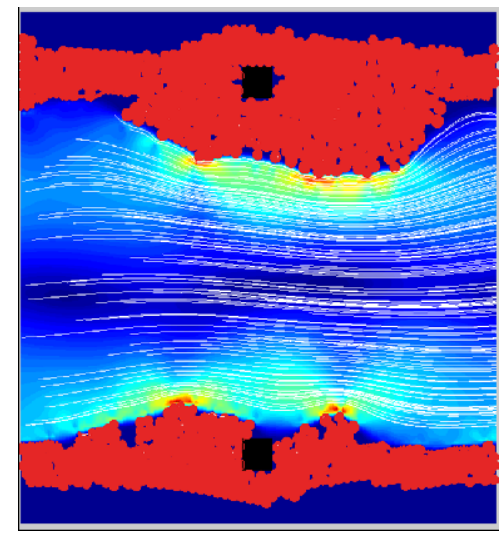

Fig. 5. Results with a drug eluting stent after 400 time steps ( $\sim 18$ days)

drug concentration (of particular note is the inhibitory effect observed when a drug eluting stent is simulated). The smooth muscle proliferation currently observed appears to share more in common with tumour cell growth, than the slower, more regulated hyperplastic response we would expect to see in a coronary artery following stent deployment. We are currently in the process of running more extended simulations and validating the model against a biological data-set obtained from stented porcine arteries. As parameters are tuned using archival quantitative data (from the literature), and experimental measurements, we hope to reproduce the positive correlation between neointimal growth rate and injury index. The next step will be to run a full $3 \mathrm{D}$ version of the model, enabling the influence of the stent geometry on the restenosis response to be investigated.

\section{Conclusions}

We have shown how Complex Automata methodology can be applied in a challenging multiscale model of in-stent restenosis. In particular, we described a conceptual multiscale model of in-stent restenosis, implementing the coupling of single scale algorithms for three different subprocesses with different time scales. The model has been realised employing a dedicated coupling library. The preliminary results demonstrate that the $\mathrm{CxA}$ model can be successfully implemented within this framework.

The individual models are at a relatively early stage of development and require further improvement. During the lifetime of the project we aim to achieve integration of a parallel flow solver (to achieve more detailed local hydrodynamics) and enhancement of the complexity of the SMC Agent rule-set. Moreover, the current 3-box CxA model might be improved further by including additional 
processes modelling thrombus formation and endothelial cell loss/re-growth. Validation of the improved and additional individual models using quantitative data derived from in vivo and in vitro experimentation will allow assessment of model accuracy, thus indicating the limitations of the models.

This first realisation of the coupled CxA is an important milestone on the journey towards a full multiscale model of in-stent restenosis, the subject of ongoing research.

Acknowledgments. This research is supported by the European Commission, through the COAST project (www.complex-automata.org, EU-FP6-IST-FET Contract 033664).

\section{References}

1. Evans, D., Lawford, P., Gunn, J., Walker, D., Hose, R., Smallwood, R., Chopard, B., Krafczyk, M., Bernsdorf, J., Hoekstra, A.: The Application of Multiscale Modelling to the Process of Development and Prevention of Stenosis in a Stented Coronary Artery. Phil. Trans. Roy. Soc. A 366, 3343-3360 (2008)

2. Morton, A., Crossman, D., Gunn, J.: The Influence of Physical Stent Parameters upon Restenosis. Pathologie Biologie 52, 196-205 (2004)

3. Kitano, H.: Computational Systems Biology. Nature 420, 206-210 (2002)

4. Sloot, P.M.A., Hoekstra, A.G.: Multiscale Modeling in Computational Biology. Accepted for publication in Briefings in Bioinformatics (2009)

5. Hoekstra, A.G., Lorenz, E., Falcone, J.-L., Chopard, B.: Towards a Complex Automata Formalism for Multi-Scale Modeling. Int. J. Mult. Comp. Eng. 5, 491-502 (2007)

6. Southern, J., Pitt-Francis, J., Whiteley, J., Stokeley, D., Kobashi, H., Nobes, R., Kadooka, Y., Gavaghan, D.: Multi-scale Computational Modelling in Biology and Physiology. Prog. Biophys. and Mol. Biol. 96, 60-89 (2008)

7. Hoekstra, A.G., Lorenz, E., Falcone, J.L., Chopard, B.: Towards a Complex Automata Framework for Multi-Scale Modeling: Formalism and the Scale Separation Map. In: Shi, Y., van Albada, G.D., Dongarra, J., Sloot, P.M.A. (eds.) ICCS 2007. LNCS, vol. 4487, pp. 922-930. Springer, Heidelberg (2007)

8. Hoekstra, A.G., Falcone, J.-L., Caiazzo, A., Chopard, B.: Multi-scale modeling with cellular automata: The complex automata approach. In: Umeo, H., Morishita, S., Nishinari, K., Komatsuzaki, T., Bandini, S. (eds.) ACRI 2008. LNCS, vol. 5191, pp. 192-199. Springer, Heidelberg (2008)

9. Chopard, B., Droz, M.: Cellular Automata Modeling of Physical Systems. Cambridge University Press, Cambridge (1998)

10. Chopard, B., Falcone, J.-L., Razakanirina, R., Hoekstra, A.G., Caiazzo, A.: On the Collision-Propagation and Gather-Update Formulations of a Cellular Automata Rule. In: Umeo, H., Morishita, S., Nishinari, K., Komatsuzaki, T., Bandini, S. (eds.) ACRI 2008. LNCS, vol. 5191, pp. 144-251. Springer, Heidelberg (2008)

11. Hegewald, J., Krafczyk, M., Tölke, J., Hoekstra, A.G., Chopard, B.: An AgentBased Coupling Platform for Complex Automata. In: Sloot, P.M.A., et al. (eds.) ICCS 2008, Part II. LNCS, vol. 5102, pp. 227-233. Springer, Heidelberg (2008) 
12. The COAST muscle (MUltiScale Coupling Library and Environment). Sources and Documentation, http://coast-dscl.berlios.de

13. Succi, S.: The Lattice Boltzmann Equation for Fluid Dynamics and Beyond. Oxford University Press, Oxford (2001)

14. Stahl, B., Evans, D., Lawford, P. and Hose, R. (in preparation) (2009)

15. Hwang, C.-W., Wu, D., Edelman, E.R.: Physiological Transport Forces Govern Drug Distribution for Stent-Based Delivery. Circulation 104, 600-605 (2001)

16. Levin, A.D., Vukmirovic, N., Hwang, C.-W., Edelman, E.R.: Specific Binding to Intracellular Proteins Determines Arterial Transport Properties for Rapamycin and Paclitaxel. PNAS 101, 9463-9467 (2004) 